\title{
Globe
}

Revue internationale d'études québécoises

\section{Penser l'histoire environnementale du Québec. Société, territoire et écologie}

\section{Stéphane Castonguay}

Volume 9, numéro 1, 2006

Penser l'histoire environnementale du Québec. Société, territoire et écologie

URI : https://id.erudit.org/iderudit/1000795ar

DOI : https://doi.org/10.7202/1000795ar

Aller au sommaire du numéro

Éditeur(s)

Globe, Revue internationale d'études québécoises

ISSN

1481-5869 (imprimé)

1923-8231 (numérique)

Découvrir la revue

Citer ce document

Castonguay, S. (2006). Penser l'histoire environnementale du Québec. Société, territoire et écologie. Globe, 9(1), 11-16. https://doi.org/10.7202/1000795ar d'utilisation que vous pouvez consulter en ligne.

https://apropos.erudit.org/fr/usagers/politique-dutilisation/ 


\title{
Introduction \\ Penser l'histoire environnementale du Québec. Société, territoire et écologie ${ }^{1}$
}

\author{
Stéphane Castonguay \\ Chaire de recherche du Canada en histoire \\ environnementale du Québec
}

Centre interuniversitaire d'études québécoises Université du Québec à Trois-Rivières

Ce numéro thématique a comme point de départ une conférence tenue au Musée McCord à Montréal en septembre 2005 et intitulée “ Positionner le Québec dans l'histoire environnementale mondiale". Organisée par le Programme d'études sur le Québec de l'Université McGill, la Chaire de recherche du Canada en histoire environnementale du Québec de l'Université du Québec à Trois-Rivières et le collectif Quelques arpents de neiges, un "atelier migratoire " du nord-est de l'Amérique, la conférence réunissait des historiens, des géographes, des anthropologues, des sociologues, des politologues et des spécialistes des sciences naturelles et des études urbaines. Les objectifs étaient d'intéresser les chercheurs québécois à l'histoire environnementale, de promouvoir l'étude de l'histoire environnementale du Québec au Québec et à l'étranger, et d'intégrer les travaux en études québécoises dans une histoire environnementale comparative.

1. La revue Globe représentait un lieu on ne peut plus approprié pour donner suite à une conférence qui cherchait à engager un discours à la fois interdisciplinaire et international. En nous invitant à publier un dossier en ses pages, les directeurs et le comité de rédaction de Globe nous permettent d'atteindre les objectifs ambitieux de cet événement. Nous les en remercions vivement.

Stéphane Castonguay, - Introduction. Penser l'histoire environnementale du Québec. Société, territoire et écologie ", Globe. Revue internationale d'études québécoises, vol. 9, no 1, 2006. 
Cette conférence conviait des spécialistes de l'étude du Québec - de son histoire, de sa société, de son territoire et de son écologie - à engager un dialogue propre à favoriser l'adoption explicite d'un cadre d'analyse en histoire environnementale. La conférence devait ainsi fournir un forum critique pour l'introduction d'idées encore peu connues ou dont la circulation était inhibée par les cloisons disciplinaires de l'univers académique.

Parce qu'elle juxtaposait des expériences nationales et internationales, la conférence invitait à une réflexion sur les jeux d'échelles et, comme le veut le dicton écolo, à "agir localement et penser globalement ". L'action dont il est question ici est bien celle de la pratique universitaire, et non pas le militantisme qui a pu marquer à ses débuts le champ de l'histoire environnementale. En effet, si l'histoire environnementale s'est développée, en Amérique du Nord, dans la foulée des contestations environnementales, elle a cessé de limiter ses analyses à la dénonciation des perturbations et des atteintes à la * nature " pour questionner la notion même de nature, pour identifier les inégalités qui découlent de l'accès à - et de l'usage de - ressources naturelles, pour analyser, enfin, comment la nature et la culture s'imbriquent pour constituer des nouvelles formes de pouvoir ${ }^{2}$.

Considérant la richesse et l'intensité de la recherche en histoire environnementale aux États-Unis et en Europe, ainsi que l'intérêt de présenter les multiples travaux poursuivis à travers le monde, la conférence visait à renouveler l'historiographie sur l'exploitation des ressources naturelles, les transformations du paysage, l'urbanisation et l'industrialisation, ainsi que les modes d'occupation du territoire. S'il s'agit là de thèmes abondamment traités en histoire et en géographie au Québec, il faut bien comprendre que l'histoire environnementale - telle que la pratique une communauté internationale de chercheurs - suggère l'emploi de nouvelles problématiques, de nouvelles sources et de nouvelles méthodes pour interpréter un phénomène aussi fondamental que celui du rapport de la société à son environnement. Prenons l'industrialisation,

2. Simon SchamA, Landscapes and Memory, New York, Vintage Books, 1995; Richard WhITE, The Organic Machine, New York, Hill and Wang, 1995. 
phénomène analysé dans ses facettes les plus diverses par les spécialistes des sciences humaines et sociales. L'historien américain Ted Steinberg a démontré que l'établissement des filatures dans la vallée de la rivière Merrimack, au Massachusetts, berceau de l'industrialisation en Amérique du Nord, fut accompagné non seulement d'une transformation du mode de production et des structures sociales, mais aussi de la mise en place d'un nouveau mode d'exploitation de l'environnement ${ }^{3}$. Or, cette mainmise sur l'environnement contribuait tout aussi bien que la propriété des moyens de production à encadrer et à contrôler les populations laborieuses de Lowell et des environs. De même, l'histoire de l'environnement urbain en Europe et en Amérique mène à une relecture des rapports entre la ville et la campagne conduisant à dégager l'empreinte écologique des phénomènes d'urbanisation. Cette relecture, qui s'appuie aussi bien sur les matières que la ville retourne à la campagne que sur les ressources que la ville draine de la campagne pour accroitre sa richesse et son ascendant sur cette même campagne, jette un nouvel éclairage sur les dimensions environnementales de la séparation des sphères de production et de consommation ${ }^{4}$.

Outre la présentation des recherches en histoire environnementale, la conférence cherchait à combiner des thématiques environnementales à des enjeux de l'histoire du Québec et à apprécier la valeur heuristique de cette intégration. Considérons l'histoire de la forêt au Québec. Après l'analyse et la comparaison des diverses hypothèses sur l'intégration des activités agricoles et forestières et leur impact sur la socioéconomie et le développement des régions $s^{5}$, après l'étude du rôle de l'industrie

3. Theodore STEINBERG, Nature Incorporated. Industrialization and the Waters of New England, Ahmerst, University of Massachusetts Press, 1994.

4. William CRONON, Nature's Metropolis. Chicago and the Great West, New York, Norton, 1989; Steven STou,, The Fruits of Natural Advantages. Making the Industrial Countryside in California, Berkeley, University of California Press, 1998 ; Sabine BARIEs, L'invention des déchets urbains. France 1790-1970, Seyssel, Champs Vallon, 2005.

5. Comparons Jack LITTLE, Colonialism, Nationalism and Capitalism. The Upper St. Francis, Montréal/Kingston, McGill-Queen's University Press, 1988 ; Normand SÉguin, La conquête du sol au XIX siècle, Montréal, Boréal Express, 1977 ; Guy GAUDREAU, * Le rapport agriculture-forêt au Québec ", Retue d'bistoire de l'Amérique française, vol. 33, $\mathrm{n}^{\circ} 1,1979$, p. 67-78; Gérard BoucHARD, * Co-intégration et 
papetière dans l'évolution du capitalisme monopolistique au début du $\mathrm{xx}^{\mathrm{e}}$ siècle $^{6}$, une histoire socio-économique de la forêt québécoise renouvellerait ses problématiques en tablant sur les travaux récents en histoire environnementale. Qu'il s'agisse de l'accès et du contrôle des réserves forestières et des parcs naturels ${ }^{7}$ ou des dynamiques écologiques soustendant les phénomènes de reboisement et $d^{\prime}$ 'incendie ${ }^{8}$, l'histoire environnementale a multiplié les facettes de la forêt et montré comment les rapports sociaux à la forêt s'appuient sur différentes représentations de la nature et donnent lieu à différents modes de régulation sociale 9 . De même, l'histoire urbaine, maintes fois campée dans l'analyse de l'organisation spatiale des inégalités sociales, voit se multiplier les éléments de ces inégalités pour peu que nous nous intéressions aux enjeux environnementaux de la ville comme les inondations ${ }^{10}$, les pollutions industrielles ${ }^{11}$, ou les animaux et leurs déchets en milieu urbain ${ }^{12}$.

reproduction de la société rurale. Pour un modèle saguenayen de la marginalité ", Recherches sociograpbiques, vol. 29, $\mathrm{n}^{\text {os }} 2-3,1988$, p. 283-310.

6. Pensons aux travaux de Gilles PIÉDalue, * Les groupes financiers et la guerre du papier au Canada 1920-1930", Revue d'bistoire de l'Amérique française, vol. $30, \mathrm{n}^{\circ} 2$, septembre 1976, p. 223-258; Jorge NIOSI, * La Laurentide (1887-1928). Pionnière du papier journal au Canada ", Revue d'bistoire de l'Amérique française, vol. $29, \mathrm{n}^{\circ} 3,1975$, p. $375-415 ;$ Jean-Pierre ChARLAND, Les pâtes et papiers au Québec 1880-1980. Technologies, travail et travailleurs, Québec, Institut québécois de recherche sur la culture, 1990.

7. Nancy Peuuso, Rich Forest, Poor People, Berkeley, University of California Press, 1992; Karl JACOBY, Crimes Against Nature. Squatters, Poachers, and the Hidden History of American Conservation, Los Angeles, University of California Press, 2001

8. Nancy Langston, Forest Dreams, Forest Nightmares. The Paradox of old Growth in the Inland West, Seattle, University of Washington Press, 1995 ; Stephen J. PYNE, Fire in America. A Cultural History of Wildland and Rural Fire, Seattle, University of Washington Press, 1997; Marcus HaLl, "Repairing Mountains. Restoration, Ecology, and Wilderness in Twentieth-Century Utah *, Environmental History, vol. 6, octobre 2001, p. 584-610; Emily Brock, "The Challenge of Reforestation. Ecological Experiments in the Douglas Fir Forest, 1920-1940 •, Environmental History, vol. 9, n 1, janvier 2004, p. 57-79.

9. Stéphane CASTONGUAY, a Foresterie scientifique et reforestation. L'État et la production d'une "forêt à pâte" au Québec", Revue d'bistoire de l'Amérique française, vol. $60, \mathrm{n}^{\circ \mathrm{s}} 1-2,2006, \mathrm{p} .61-93$.

10. Christopher G. BOONE, "Language Politics and Flood Control in NineteenthCentury Montreal ", Environmental History, vol. 1, no 3, 1996, p. 70-85.

11. Voir les contributions dans l'ouvrage collectif dirigé par Christoph BERNHARDT et Geneviève MASSARD-GuILAud (éd.), Le démon moderne. La pollution dans les 


\section{INTRODUCTION}

De tous les thèmes de la conférence, nous en avons retenu cinq pour sélectionner les articles ici colligés : les approches en histoire environnementale, l'impérialisme et les problèmes environnementaux globaux, l'occupation du territoire nordique et les Premières nations, les dynamiques des écosystèmes, l'environnement urbain et l'industrialisation $^{13}$. Regroupés en deux grandes familles, celle de l'historiographie et celle de la monographie, ces articles nous permettent d'explorer les avenues récentes de l'histoire environnementale, de réfléchir sur le potentiel du Québec comme objet d'études pour le développement de l'historiographie de l'histoire environnementale, et d'animer une réflexion sur les problèmes environnementaux à l'intérieur d'un discours historien.

Les premiers articles dressent des bilans de l'histoire du Québec, de l'histoire de l'Amérique du Nord en général et de sa côte atlantique en particulier, ainsi que des études urbaines. D'abord, Stéphane Castonguay démontre la pertinence des travaux en études québécoises pour les praticiens de l'histoire environnementale, qui y trouveront à la fois des sources et des questionnements apparentés à ses problématiques. Puis Donald Worster précise les dimensions environnementales du récit historique et signale le potentiel de l'histoire environnementale pour renouveler notre compréhension du passé. Remarquant l'influence prépondérante de l'expérience de la transformation environnementale de l'Ouest américain dans la conscience environnementaliste et la constitution du champ de l'histoire environnementale, Richard Judd s'interroge sur la spécificité de l'expérience du territoire et de la population de la côte est de l'Amérique du Nord, négligée lors du démarrage de l'histoire environnementale, et en tire des enseignements pertinents pour l'étude du Québec. Enfin, dans un état des lieux en histoire

sociêtés urbaines et industrielles d'Europe/The Modern Demon. Pollution in Urban and Industrial European Societies, Clermont-Ferrand, Presses de l'UBP, coll. * Histoires croisées ", 2002.

12. Christopher OTTER, "Cleansing and Clarifying. Technology and Perception in Nineteenth-Century London ", Journal of British Studies, vol. 43, $\mathrm{n}^{\circ} 1$, janvier 2004, p 40-64.

13. On trouvera le programme complet sur le site de la Chaire de recherche du Canada en histoire environnementale du Québec, http://www.cieq.uqtr.ca/ crc_hec/activites_conf.php (1 ${ }^{\text {er }}$ décembre 2006). 


\section{REVUE INTERNATIONALE D'ÉTUDES QUÉBÉCOISES}

environnementale et en études urbaines au Québec, Claire Poitras présente les dimensions et les enjeux environnementaux de la ville et propose des chantiers pour le développement de l'histoire urbaine environnementale au Québec.

Les cinq derniers articles illustrent la portée des questionnements de l'histoire environnementale pour les spécialistes des différentes disciplines en études québécoises. Sociologue et spécialiste des recherches amérindiennes, Denys Delâge inscrit l'étude des rapports sociaux à la nature des populations amérindiennes dans des problématiques telles que l'échange microbien ou les rapports à l'eau et aux animaux. Spécialiste de l'histoire des feux à travers le monde, Stephen Pyne fonde son étude du Québec sur les incendies de forêt et les institutions dont se dota le gouvernement provincial non seulement pour tenter de contrôler ce phénomène écologique, mais aussi pour résoudre des tensions sociales et politiques touchant l'accès et la protection de la ressource forestière. La géographe Caroline Desbiens aborde l'histoire de la rivière Chisasibi/La Grande et de ses usages par les populations cries et québécoises à travers le prisme des rapports d'échange et de force entre le sud et le nord. Enfin, les politologues Carole Beaudoin, David Houle et Jean Mercier évaluent les politiques du ministère de l'Environnement du Québec des trente premières années sous l'angle de la " nouvelle gouvernance ". Se greffe à ces quatre études une réflexion synthétique sur la " fabrication d'une nouvelle histoire * par un spécialiste de l'histoire sociale et économique du Québec, Jean-Claude Robert, dont les propos clôturaient la conférence "Positionner le Québec dans l'histoire environnementale mondiale ". 